\title{
ESTIMATIVAS VOLUMÉTRICAS EM PLANTIOS DE EUCALIPTO PARA DUAS MESORREGIÕES DO RIO GRANDE DO SUL
}

\section{VOLUME ESTIMATES IN EUCALYPTUS PLANTATIONS FOR TWO MESOREGIONS OF RIO GRANDE DO SUL}

\author{
Matheus Teixeira Martins ${ }^{1}$, Emanuel Arnoni Costa ${ }^{2}$, Gabriel Paes Marangon ${ }^{3}$ \\ 1,3 Universidade Federal do Pampa, São Gabriel, RS, Brasil - mteixeiramartins@gmail.com \& \\ gabrielmarangon@unipampa.edu.br
}

${ }^{2}$ Universidade Federal de Uberlândia, Monte Carmelo, MG, Brasil - emanuelarnonicost@hotmail.com

\section{RESUMO}

O volume de árvores é a variável de maior interesse florestal, sendo possível estima-la por várias técnicas, sendo a modelagem a mais usual. Portanto, o presente estudo teve como objetivo ajustar, validar e selecionar o melhor modelo volumétrico para árvores de Eucalyptus grandis W. Hill. ex Maiden de plantios em duas mesorregiões do estado do Rio Grande do Sul. Para isso, 791 árvores foram cubadas pelo método da Smalian e separadas em grupos de ajuste ( $\approx 74 \%)$ e validação ( $\approx 26 \%)$. Três modelos foram testados: (Husch) $v=\beta_{0} \times$ dap $^{\beta 1} \times \varepsilon_{i}$; (Schumacher e Hall) $v$ $=\beta_{0} \times$ dap ${ }^{\beta 1} \times \mathrm{h}^{\beta 2} \times \varepsilon_{\mathrm{i}}$; (Schumacher e Hall modificado) $\mathrm{v}=\beta_{0} \times$ dap $^{\beta 1} \times \mathrm{h}^{\beta 2} \times \mathrm{h}_{100^{\beta 3}} \times \varepsilon_{\mathrm{i}}$. Os modelos foram avaliados de acordo com os seguintes critérios: coeficiente de determinação ajustado ( $R^{2}$ aj.), raiz quadrada do quadrado médio do erro em porcentagem (RMSE\%) e análise gráfica dos resíduos em porcentagem (E\%). De acordo com o teste $\chi^{2}$ à 5 $\%$ de probabilidade, não houve diferença significativa entre as estimativas volumétricas para as três equações com e sem casca quando confrontados com os volumes reais. O modelo de Schumacher e Hall modificado apresentou melhores resultados e tem maior destaque por utilizar a variável de altura dominante de Assmann $\left(h_{100}\right)$ que caracteriza o sítio florestal e com possibilidade de ampla utilização.

PALAVRAS-CHAVE: Cubagem, Eucalyptus grandis, Florestas plantadas, Inventário florestal, Volumetria.

\section{ABSTRACT}

The volume of trees is the variable of greatest forest interest, being possible to estimate it by various techniques, with modeling being the most usual. Therefore, the present study aimed to adjust, validate and select the best volumetric model for Eucalyptus grandis W. Hill. ex Maiden from plantations in two mesoregions of the state of Rio Grande do Sul. For that, 791 trees were cut by the Smalian method and separated into adjustment $(\approx 74 \%)$ and validation ( $\approx 26 \%$ ) groups. Three models were tested: (Husch) $v=\beta_{0} \times$ dap $^{\beta 1} \times \varepsilon_{i}$; (Schumacher and Hall) $v=\beta_{0} \times$ dap $^{\beta 1}$ $\times h^{\beta 2} \times \varepsilon_{i}$; (Schumacher and Hall modified) $v=\beta_{0} \times$ dap $^{\beta 1} \times h^{\beta 2} \times h_{100^{\beta 3}} \times \varepsilon_{\mathrm{i}}$. The models were evaluated according to the following criteria: adjusted determination coefficient $\left(R^{2}\right.$ aj.), Square root of the mean square of the error in percentage (RMSE\%) and graphic analysis of the residuals in percentage (E\%). According to the $\chi 2$ test at $5 \%$ probability, there was no significant difference between the volumetric estimates for the three equations with and without shell when compared to the actual volumes. The modified Schumacher and Hall model showed better results and is more prominent for using the variable of dominant height of Assmann $\left(\mathrm{h}_{100}\right)$ that characterizes the forest site and with the possibility of wide use.

KEYWORDS: Cubage, Eucalyptus grandis, Planted forests, Forest inventory, Volumetry. 


\section{INTRODUÇÃO}

O Brasil é destaque na produção de florestas plantadas, compostas principalmente por eucalipto, espécie exótica, nativa da Austrália, que se adaptou facilmente às condições climáticas do país, apresentando fácil manejo e rápido crescimento. A produção industrial é o principal uso do eucalipto no Brasil, sendo fonte de matéria-prima para as indústrias de celulose e papel (MIRANDA et al., 2015).

Em 2018, a produtividade média dos plantios de eucalipto no país foi de $36 \mathrm{~m}^{3} /$ ha.ano, estando a frente da China, Estados Unidos, Rússia, Canadá, entre outros países. O estado do Rio Grande do Sul apresentou 308.657 ha de área ocupada por plantios de eucalipto em 2018, equivalendo a $5 \%$ de área total do estado. Neste quesito o estado do Rio Grande do Sul se apresenta com menor ocupação quando comparado aos estados de Minas Gerais com $24 \%$, São Paulo com $17 \%$, Mato Grosso do Sul com 16\% e Bahia com 11\% (IBÁ, 2019).

O manejo técnico de uma floresta para otimizar o uso de recursos econômicos e ecológicos exige, como ponto de partida para a tomada de decisão, a disponibilidade de dados qualitativos e quantitativos da floresta, o que resulta em informações sobre o volume de madeira por espécie e por hectare, estoque de regeneração natural, entre outros. Portanto, a dendrometria é de fundamental importância na geração de informações sempre que qualquer projeto ou atividade florestal é estabelecido (CUNHA, 2004).

No inventário florestal, uma das principais funções do ponto de vista econômico é estimar o estoque de madeira da floresta e sua produção para direcionar a matéria-prima para seu uso determinado (GAROSI et al., 2008).

O volume é uma das variáveis de maior destaque na floresta, pois a partir dele se tem um ponto de inicial para avaliar o potencial produtivo e o estoque de madeira que será fornecido pela floresta. Há diferentes métodos de obter o volume, como, a cubagem, estimativas por equações volumétricas, fatores de forma, funções de afilamento, entre outros.

$\mathrm{O}$ método de cubagem rigorosa é bastante oneroso e caro, portanto, a sua aplicação é aliada aos demais métodos, para que assim se tenha redução do número de árvores cubadas e seja possível estimar o volume das demais. Na literatura são encontrados diversos estudos, voltados ao uso dos métodos de obtenção de volume de árvores de eucalipto, como relatado por: Azevedo et al. (2011), que testaram modelos volumétricos visando selecionar o mais acurado; Leal et al. (2015), ajustaram e validaram modelos volumétricos para árvores de eucalipto visando à redução do número de árvores cubadas; Miranda et al. (2015), desenvolveram um fator de forma e uma equação de volume para estimar o volume comercial e total de árvores; Binoti et al. (2014), desenvolveram uma metodologia de aplicação de RNA para estimar o volume de árvores, visando reduzir o número de indivíduos a serem cubados; Silva et al. (2016), avaliaram o uso de regressão e RNA para estimar o afilamento de fustes de eucalipto em sistemas silvipastoris; Téo et al. (2018), ajustaram e selecionaram modelos de afilamento para estimar diâmetros, volume por seção e volume acumulado com casca, para árvores de eucalipto; entre outros estudos.

A comercialização de produtos florestais desempenha um papel importante no desenvolvimento socioeconômico do país, portando é indispensável a realização de estudos que avaliem as maneiras de obtenção de estimativas relacionadas às florestas plantadas, principalmente no que diz respeito ao conhecimento do estoque de madeira (MIGUEL et al., 2010).

Diante do exposto, o objetivo do estudo foi ajustar e validar equações para estimar o volume total com e sem casca de árvores em plantios de Eucalyptus grandis W. Hill ex Maiden em diferentes mesorregiões do estado do Rio Grande do Sul e, assim, obter a equação que gera as melhores estimativas para as condições da área de estudo.

\section{MATERIAL E MÉTODOS}

\section{Caracterização e localização da área}

Para a realização do estudo, foram utilizados dados de plantios adensados de $E$. grandis em diferentes idades e localizadas em duas mesorregiões do estado do Rio Grande do Sul, nos munícipios de Santa Cruz do Sul, Lajeado, Estrela e Cachoeira do Sul (mesorregião Centro Oriental Rio-grandense) e Montenegro, Gramado, Canela, Porto Alegre, Osório e Camaquã (mesorregião Metropolitana).

O clima das regiões estudadas de acordo com a classificação climática de Köppen é do tipo Cfa, com temperatura média anual de $17,9{ }^{\circ} \mathrm{C}$ e precipitação média anual de $1.826 \mathrm{~mm}$ (ALVARES et al., 2013).

Os solos pertencem à unidade de mapeamento Rio Pardo, sendo classificados como argissolos vermelhos distróficos plintossólicos, que são profundos, de coloração avermelhada em toda a extensão do perfil, argilosos, bem drenados e derivados de siltitos finos (FEPAM, 2001; EMBRAPA, 2018).

\section{Amostragem dos dados}


Os dados são provenientes do inventário florestal com parcelas de $30 \mathrm{~m} \times 20 \mathrm{~m}\left(600 \mathrm{~m}^{2}\right)$ em plantios com diferentes espaçamentos iniciais $(1,0 \mathrm{~m} \times 1,5 \mathrm{~m} ; 3,0 \mathrm{~m} \times$ $1,7 \mathrm{~m} ; 2,3 \mathrm{~m} \times 2,3 \mathrm{~m} ; 2,0 \mathrm{~m} \times 2,0 \mathrm{~m}$, entre outros) e com idade entre 10 e 30 anos.

O banco de dados é formado por árvores médias mensuradas e cubadas em campo formando um conjunto de 791 observações, com as seguintes variáveis: altura total $(\mathrm{h})$, altura dominante de Assmann $\left(\mathrm{h}_{100}\right)$, diâmetro à altura do peito (dap), volume com casca ( $\left.v_{c c}\right)$ e volume sem casca $\left(v_{s c}\right)$. Desse conjunto de dados, 582 ( $\left.\approx 74 \%\right)$ observações foram separadas para o ajuste das equações volumétricas e 209 ( $\approx 26 \%$ ) observações para a validação dessas equações. A separação dos dados nesses dois grupos foi realizada de forma aleatória, porém, abrangendo as classes diamétricas dos indivíduos, resultando em uma distribuição uniforme dos dados.

\section{Análise dos dados}

Foram selecionados três modelos volumétricos (Tabela 1) consagrados na literatura para realização do ajuste.

Tabela 1. Modelos ajustados para estimar o volume das árvores de plantios de $E$. grandis.

\begin{tabular}{|c|c|c|}
\hline Modelo & Expressão & Autores \\
\hline 1 & $v=\beta_{0} \times \operatorname{dap}^{\beta 1} \times \varepsilon_{i}$ & Husch \\
\hline 2 & $v=\beta_{0} \times \operatorname{dap}^{\beta 1} \times \mathrm{h}^{\beta 2} \times \varepsilon_{\mathrm{i}}$ & Schumacher e Hall \\
\hline 3 & $v=\beta_{0} \times \operatorname{dap}^{\beta 1} \times h^{\beta 2} \times h_{100}{ }^{\beta 3} \times \varepsilon_{i}$ & $\begin{array}{c}\text { Schumacher e Hall } \\
\text { (Modificado) }\end{array}$ \\
\hline
\end{tabular}

Em que: $\beta_{0}, \beta_{1}, \beta_{2}$ e $\beta_{3}$ - coeficientes de regressão estimados; $v$ volume com e sem casca, em $\mathrm{m}^{3}$; dap - diâmetro à altura do peito, em $\mathrm{cm} ; \mathrm{h}$ - altura total, em $\mathrm{m} ; \mathrm{h}_{100}$ - altura dominante de Assmann, em $\mathrm{m} ; \varepsilon_{\mathrm{i}}$ - erro aleatório.

Para verificar a eficiência das equações foram usados os seguintes critérios estatísticos, o coeficiente de determinação ajustado $\left(R^{2}\right.$ aj.), a raiz quadrada do erro quadrático médio em porcentagem (RMSE\%) e a análise gráfica da porcentagem de resíduos (E\%), representada pelas seguintes expressões:

$$
\begin{gathered}
R^{2} \text { aj. }=1-\left[\frac{\sum_{i=1}^{n}\left(Y_{i}-\hat{Y}_{i}\right)^{2}}{\sum_{i=1}^{n}\left(Y_{i}-\bar{Y}\right)^{2}}\right] \times\left[\frac{(n-1)}{(n-p)}\right] \\
\text { RMSE\% }=\frac{\sqrt{(n-p)^{-1} \sum_{i=1}^{n}\left(Y_{i}-\hat{Y}_{i}\right)^{2}}}{\bar{Y}} \times 100 \\
E \%=\frac{\left(Y_{i}-\hat{Y}_{i}\right)}{Y_{i}} \times 100
\end{gathered}
$$

Em que: $Y_{i}$ - volume observado, em $\mathrm{m}^{3} ; \widehat{\mathrm{Y}} \mathrm{i}$ - volume estimado, em $\mathrm{m}^{3} ; \overline{\mathrm{Y}}$ - média do volume observado, em $\mathrm{m}^{3} ; \mathrm{n}$ - número de observações; $p$ - número de coeficientes do modelo.
Após a obtenção dos coeficientes de cada modelo, foi realizada a aplicação dos mesmos ao conjunto de validação. Para verificar a magnitude da eficiência das estimativas feitas para o conjunto de validação com as equações geradas no ajuste, foram calculados os critérios de $R^{2}$ aj. e RMSE\%. Além disso, foi realizado o teste QuiQuadrado $\left(\chi^{2}\right)$ à $5 \%$ de probabilidade para comparação entre os volumes reais e volumes estimados, representado pela expressão:

$$
\chi^{2}=\sum_{i=1}^{n} \frac{\left(Y_{i}-\widehat{Y_{i}}\right)^{2}}{\widehat{Y_{i}}}[4]
$$

Em que: $Y_{i}$ - volume observado para cada árvore, em $\mathrm{m}^{3} ; \widehat{Y}_{i}$ volume estimado para cada árvore, em $\mathrm{m}^{3} ; \mathrm{n}$ - número de observações.

A verificação de eficiência na validação das estimativas com a aplicação do teste $\chi^{2}$ é comumente usada na literatura (LEAL et al., 2015; OLIVEIRA et al., 2016). A manipulação dos dados foi realizada no software Microsoft Office Excel 2019 e os ajustes e estatísticas dos modelos no software $R$ versão 3.2.4 (R CORE TEAM, 2016).

\section{RESULTADOS E DISCUSSÃO}

As estatísticas descritivas dos grupos de dados usados para o ajuste e validação dos modelos volumétricos em plantios de $E$. grandis são apresentados na Tabela 2. Esses dados são provenientes de plantios de eucalipto adensados, resultando em árvores com pequeno dap e maiores alturas totais. Todos os coeficientes estimados dos três modelos volumétricos ajustados foram significativos pelo teste $t(p<0,001)$ (Tabela 3$)$.

O modelo 3 (Schumacher e Hall modificado) com e sem casca apresentou os melhores resultados estatísticos. Neste modelo, três variáveis independentes ( $h, h_{100}$ e dap) são utilizadas, obtendo estimativas mais precisas do volume das árvores, quando comparado ao modelo 1 (Husch), que utiliza apenas uma variável independente (dap) e ao modelo 2 (Schumacher e Hall) que utiliza duas variáveis independentes (dap e h). Os critérios estatísticos para o volume sem casca foram inferiores em questão de eficiência para os três modelos ajustados (Tabela 3).

É notável que há melhoria dos resultados estatísticos em modelos com maior número de variáveis independentes, o modelo 3 (Schumacher e Hall modificado) apresentou maior $\mathrm{R}^{2}$ aj. e menor RMSE\%. Porém, mesmo com melhor desempenho dos modelos de maior número de variáveis independentes, muitas vezes a obtenção das mesmas se torna onerosa, e assim, se torna mais prática a utilização de modelos volumétricos mais simples. 
Tabela 2. Estatística descritiva para as variáveis dendrométricas em plantios de $E$. grandis.

\begin{tabular}{ccccccc}
\hline Dados & Variável & Mínimo & Média & Mediana & Máxima & CV\% \\
\hline \multirow{2}{*}{ Ajuste } & dap & 10,3 & 16,3 & 16,3 & 22,1 & 13,6 \\
(582 observações) & $\mathrm{h}$ & 12,7 & 22,9 & 23,6 & 32,6 & 17,0 \\
& $\mathrm{~h}_{100}$ & 14,3 & 29,5 & 30,1 & 44,3 & 22,2 \\
& $\mathrm{v}_{\mathrm{cc}}$ & 0,0444 & 0,2653 & 0,2637 & 0,5596 & 43,7 \\
& $\mathrm{v}_{\mathrm{sc}}$ & 0,0362 & 0,2257 & 0,2240 & 0,4825 & 44,3 \\
\hline \multirow{2}{*}{ Validação } & $\mathrm{dap}$ & 10,4 & 16,2 & 16,2 & 21,3 & 14,2 \\
(209 observações) & $\mathrm{h}$ & 12,5 & 22,8 & 23,7 & 30,6 & 16,9 \\
& $\mathrm{~h}_{100}$ & 14,0 & 28,8 & 29,7 & 41,9 & 20,9 \\
& $\mathrm{v}_{\mathrm{cc}}$ & 0,0478 & 0,2568 & 0,2494 & 0,5518 & 44,0 \\
& $\mathrm{v}_{\mathrm{sc}}$ & 0,0389 & 0,2183 & 0,2119 & 0,4748 & 44,8 \\
\hline
\end{tabular}

Em que: $\mathrm{CV} \%$ = coeficiente de variação.

Os resultados estatísticos observados no modelo 1 (Husch) são semelhantes ao obtido por Azevedo et al. (2011), que constatou que o modelo utilizando apenas a variável dap para estimar o volume apresentou estatísticas menos eficientes comparado aos modelos que utilizavam mais variáveis independentes, como, a altura. Couto e Bastos (1987) afirmam que a determinação da altura de árvores é uma operação bastante onerosa e sujeita a erros e salientam que é importante à avaliação de modelos que não dependem do uso desta variável, pois assim pode haver redução de tempo e de custo no inventário florestal. Soares et al. (2007), afirmam que tanto para altura, como para dap, podem ocorrer erros de medição, porém, se tratando de dap é mais fácil controlar esse erro.

$\mathrm{O}$ ajuste do modelo 3 (Schumacher e Hall modificado), com variáveis dap, altura total e altura de Assmann, como foi realizado neste estudo, representa uma ampliação quanto ao uso. A altura dominante de Assmann é importante para indicar a capacidade de sítio e possui alta correlação com a produção total do povoamento (FINGER, 1992).

As representações gráficas dos ajustes e análises gráficas de resíduos dos três modelos para estimar o volume total com e sem casca de árvores de E. Grandis são apresentadas nas Figuras 1 e 2.

Tanto as figuras de volume com casca, como de volume sem casca, são bastante semelhantes. Há um baixo recobrimento dos valores observados pelos valores estimados com o modelo 1 (Husch) e maior amplitude residual comparado aos demais modelos. 0 modelo 2 (Schumacher e Hall) apresentou boas estimativas, recobrindo bem o conjunto de dados observados para ambos volumes avaliados e teve menor amplitude residual comparado ao modelo 1 (Husch), podendo-se dizer que foi um modelo intermediário, pois, não foi o mais eficiente quando comparado ao modelo 3 (Schumacher e Hall Modificado). Portanto, com os resultados estatísticos obtidos e análise gráfica, o modelo 3 (Schumacher e Hall Modificado) apresentou melhores estimativas para os volumes totais com e sem casca no conjunto de dados de ajuste.

A representação tridimensional (Figura 3), mostra a superfície de tendência da proporção da altura dividida pela altura dominante em função do diâmetro para os volumes com e sem casca das árvores de $E$. grandis.

Quando a árvore apresenta $\mathrm{h} / \mathrm{h}_{100}$ de $0,5 \mathrm{~m}$ e dap de 8 $\mathrm{cm}$, observou-se que terá volumes muito pequenos. Quando esse dap é mantido e a $\mathrm{h} / \mathrm{h}_{100}$ aumenta, em torno de 1,1 $\mathrm{m}$, o volume aumenta cerca de $0,1 \mathrm{~m}^{3}$. Foi observado que nem sempre a maior árvore em altura apresentará os maiores volumes e, as árvores menores em altura, podem apresentar maiores volumes quando tem maior crescimento em diâmetro.

Com o teste Qui-quadrado à $5 \%$ de probabilidade foi verificado que não houve diferença estatística significativa entre as estimativas volumétricas com e sem casca para os três modelos analisados (Tabela 4).

Ao avaliar os resultados estatísticos obtidos na validação dos modelos, observou-se que o modelo 1 (Husch) obteve os piores resultados quando comparados aos demais modelos, isso não impede a sua aplicabilidade, - mesmo apresenta usabilidade utilizando apenas a variável dap. O modelo 2 (Schumacher e Hall), obteve resultados intermediários e o modelo 3 (Schumacher e Hall modificado) com as melhores estatísticas, comprovando a sua eficiência. 
Tabela 3. Coeficientes estimados e respectivas estatísticas de qualidade de ajustes para os modelos volumétricos analisados em plantios de $E$. grandis.

\begin{tabular}{|c|c|c|c|c|c|c|c|}
\hline Modelo & Tipo & $\beta_{0}$ & $\beta_{1}$ & $\beta_{2}$ & $\beta_{3}$ & $\mathrm{R}^{2} \mathrm{aj}$. & RMSE\% \\
\hline 1 & \multirow{3}{*}{ Com casca } & 0,000038 & 3,1544 & & & 0,913 & 12,9 \\
\hline 2 & & 0,000031 & 1,7467 & 1,3165 & & 0,946 & 10,1 \\
\hline 3 & & 0,000025 & 1,9275 & 0,3251 & 0,8268 & 0,991 & 4,2 \\
\hline 1 & \multirow{3}{*}{ Sem casca } & 0,000028 & 3,2021 & & & 0,913 & 13,1 \\
\hline 2 & & 0,000023 & 1,7517 & 1,3576 & & 0,947 & 10,2 \\
\hline 3 & & 0,000018 & 1,9315 & 0,3752 & 0,8222 & 0,990 & 4,4 \\
\hline
\end{tabular}

(a)

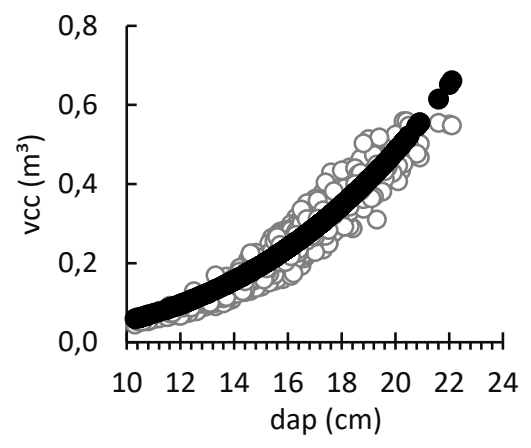

o Observado Estimado

(c)

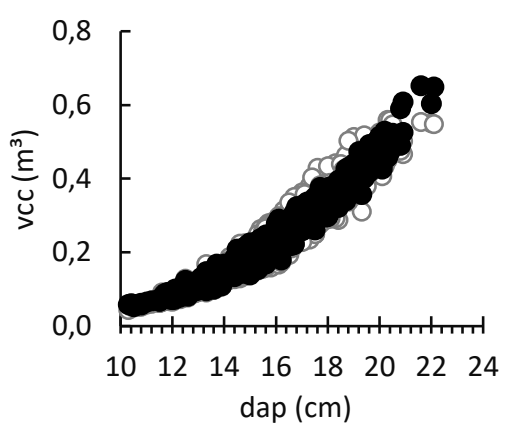

(e)

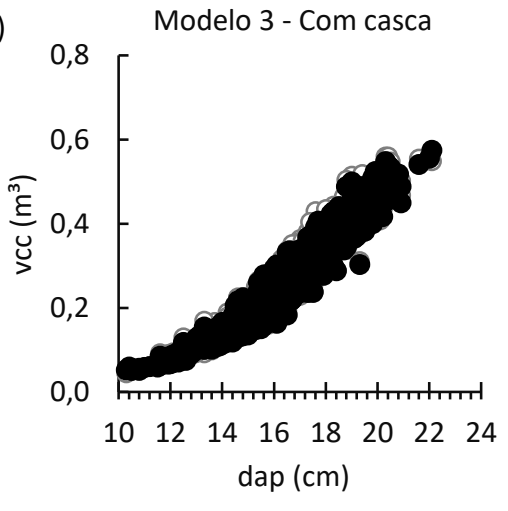

(b) Modelo 1 - Com casca

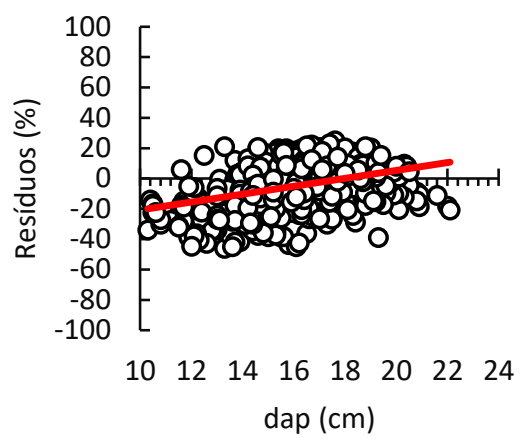

(d)

Modelo 2 - Com casca

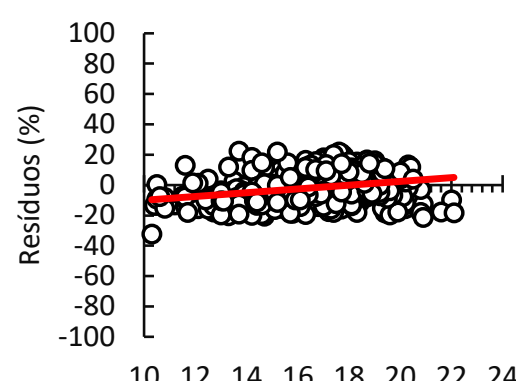

dap $(\mathrm{cm})$

(f)

Modelo 3 - Com casca

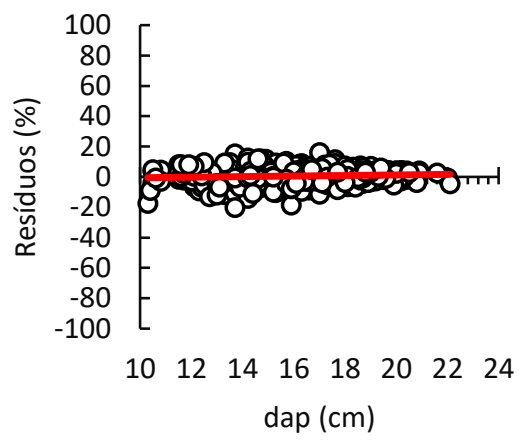

Figura 1. Tendência do volume com casca (vcc) observado e estimado (a, c e e) e análise gráfica de resíduos em porcentagem (b, d e f), ambos em função do dap para os três modelos volumétricos testados em plantios de $E$. grandis. 
(a)

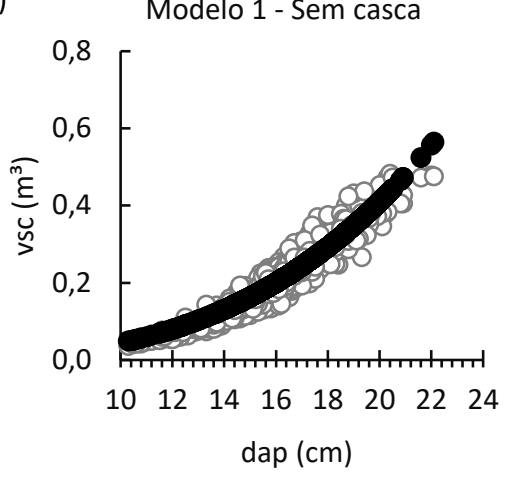

(c)

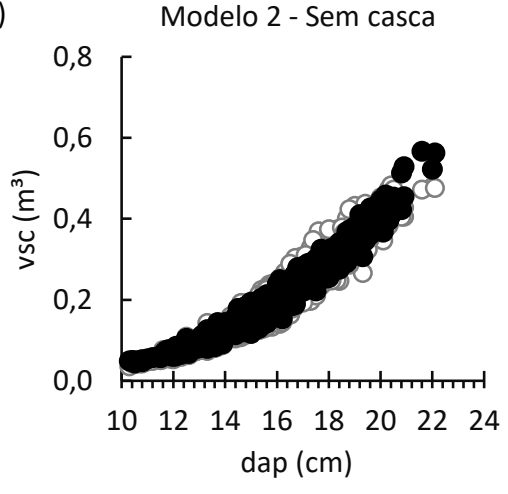

O Observado Estimado

(e)

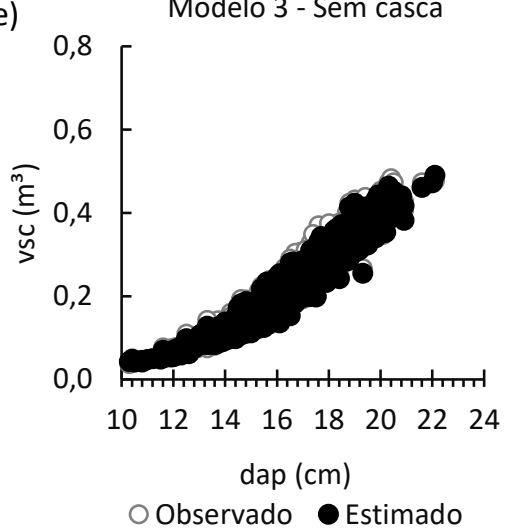

(b) Modelo 1 - Sem casca

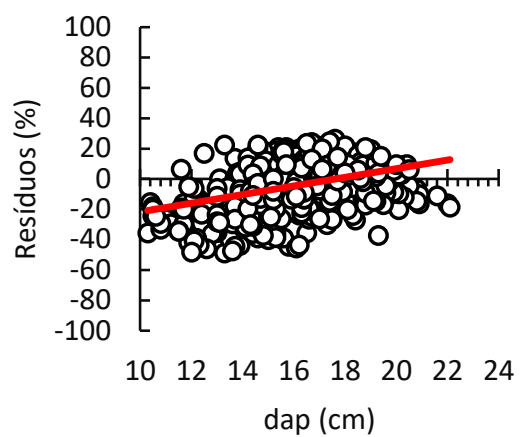

(d)

Modelo 2 - Sem casca

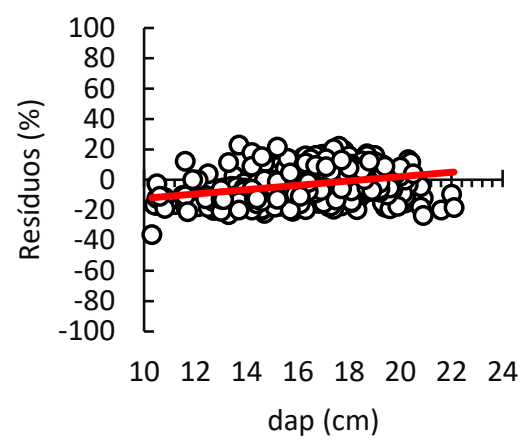

(f)

Modelo 3 - Sem casca

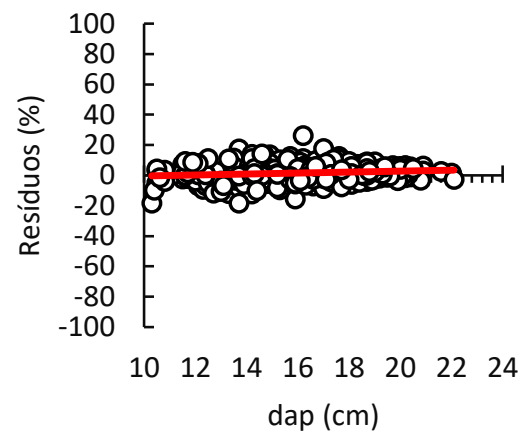

Figura 2. Tendência do volume sem casca $\left(v_{s c}\right)$ observado e estimado ( $a, c$ e e) e análise gráfica de resíduos em porcentagem (b, d e f), ambos em função do dap para os três modelos volumétricos testados em plantios de $E$. grandis.

Miguel et al. (2010), alcançaram bons resultados utilizando o modelo de Husch, porém, em sua forma logarítmica, obtendo $\mathrm{R}^{2}$ aj., de 0,98 , sendo um resultado superior ao do presente estudo, provavelmente por ter sido realizado com dados de $E$. grandis no estado de Goiás, que apresenta condições climáticas e de manejo diferentes do Rio Grande do Sul. Além disso, os autores observaram que o modelo de dupla entrada de Schumacher e Hall não foi o mais eficiente para as condições estudadas.

Na literatura é notável que o modelo de Schumacher e
Hall em suas diversas formas é bastante eficiente para estimar o volume de árvores do gênero Eucalyptus e de outros gêneros, como, Pinus. Geralmente os resultados estatísticos obtidos são bons, alcançando $R^{2}$ aj. em torno de 0,9, como descrito em Thomas et al. (2006), Azevedo et al. (2011), Sales et al. (2015), entre outros.

É recomendado o teste de vários modelos, mesmo que a espécie seja a mesma, pois as condições de sítio influenciam no comportamento do crescimento das árvores e nas estimativas de suas variáveis. 
(a)

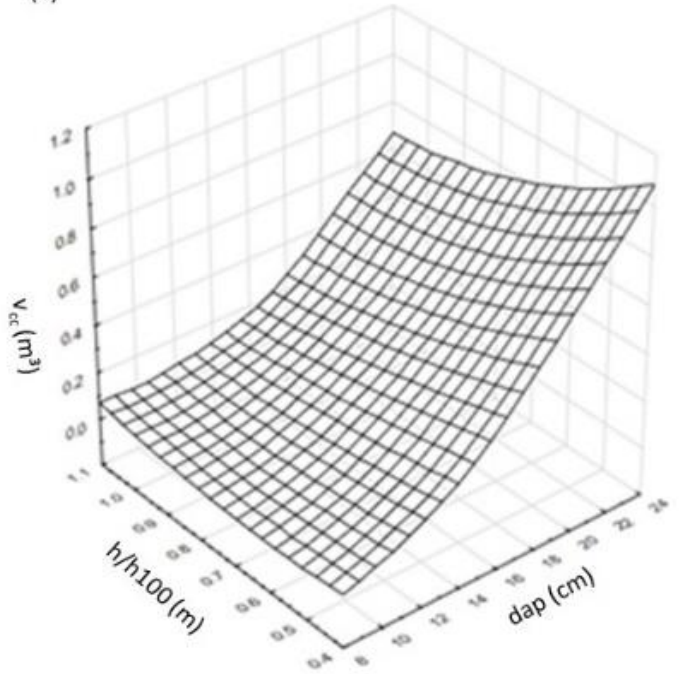

(b)

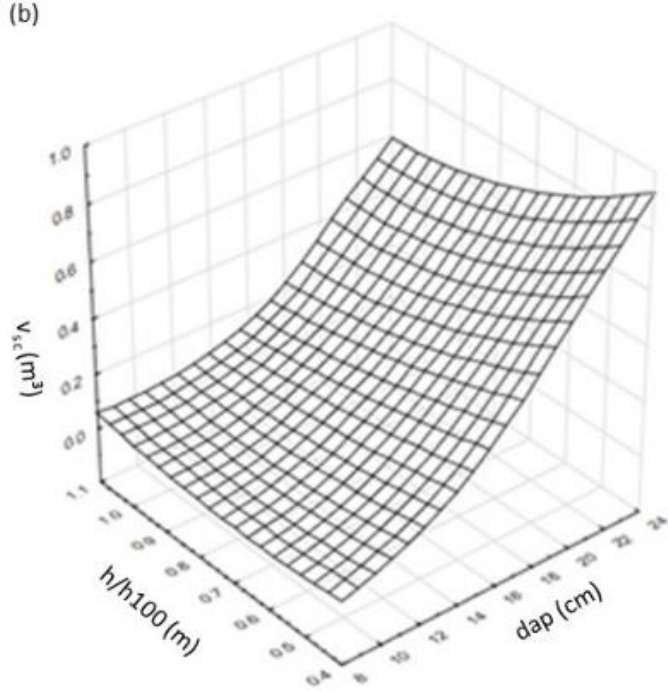

Figura 3. Representação tridimensional da relação entre a altura total ( $h$ ) dividida pela altura dominante ( $\left.h_{100}\right)$ em função do diâmetro à altura do peito $(\mathrm{dap})$ para o volume com casca $\left(\mathrm{v}_{\mathrm{cc}}\right)(\mathrm{a})$ e sem casca $\left(\mathrm{v}_{\mathrm{sc}}\right)(\mathrm{b})$ das árvores de $E$. grandis.

Tabela 4. Resultados estatísticos obtidos com a validação dos modelos para estimar o volume total com e sem casca das árvores de plantios de $E$. grandis.

\begin{tabular}{cccccc}
\hline Modelos & Tipo & $\mathbf{R}^{\mathbf{2}}$ aj. & RMSE\% & $\boldsymbol{\chi}^{\mathbf{2} \text { calculado }}$ & $\boldsymbol{\chi}^{\mathbf{2}}$ Tabelado \\
\hline $\mathbf{1}$ & \multirow{2}{*}{ Com casca } & 0,906 & 13,5 & 0,867 \\
$\mathbf{3}$ & & 0,948 & 10,0 & 0,414 \\
$\mathbf{1}$ & & 0,990 & 4,4 & 0,094 \\
$\mathbf{2}$ & \multirow{3}{*}{ Sem casca } & 0,910 & 13,4 & 0,753 \\
$\mathbf{3}$ & & 0,948 & 10,2 & 0,373 \\
\hline
\end{tabular}

O uso de modelos de dupla entrada geralmente resulta em estimativas mais acuradas, conforme relatado em Pereira et al. (2016), com destaque para o modelo de Schumacher e Hall; Miguel et al. (2010) observaram melhores performances com o modelo de Takata e destacam que este resultado era esperado, pois o volume foi estimado a partir de mais de uma variável. Também é notável que a aplicação de modelos não lineares, resulta em melhores resultados, como foi observado por: Miguel et al. (2010), Lanssanova et al. (2018), Martins et al. (2020), entre outros.

Outra técnica de regressão, que junto aos modelos não lineares geralmente é mais eficiente comparada aos modelos lineares tradicionais, são os modelos lineares generalizados. Bueno et al. (2020), relatam que esta técnica é mais flexível comparada aos modelos lineares tradicionais, pois, evita a transformação de variáveis dependentes e cálculos de erros associados.
O uso de regressão resulta em estimativas eficientes, apesar de atualmente existirem novas técnicas, como a inteligência artificial (IA), como relatado por: Cordeiro et al. (2015), Santos et al. (2018), Neto et al. (2019), entre outros. Os modelos volumétricos ainda são bastante aplicados, devido a este método de regressão tradicional ser útil e confiável para diferentes conjuntos de dados (STEPKA et al., 2017; SILVA et al., 2017; SANQUETTA et al., 2017).

Os volumes com e sem casca em função das variáveis (dap, h e h100) são apresentados na Tabela 5. Por exemplo, uma árvore, com altura de 10,9 m, dap de $16 \mathrm{~cm}$ e situada em uma parcela com $\mathrm{h}_{100}$ de $11,8 \mathrm{~m}$, tem o volume com casca de $0,0873 \mathrm{~m}^{3}$ e sem casca de 0,0708 $\mathrm{m}^{3}$. Esta é uma tabela estática e ao usar a equação de Schumacher e Hall (modificado) ajustada no presente estudo é possível prever os volumes de forma dinâmica para locais que apresentem condições semelhantes. 
Tabela 5. Tabela de volumes com e sem casca para plantios de $E$. grandis.

\begin{tabular}{|c|c|c|c|c|c|c|c|c|c|c|c|c|c|c|c|c|}
\hline$v_{c c}\left(m^{3}\right)$ & & & & & & & & & & & & & & & & \\
\hline \multirow[t]{2}{*}{$v_{s c}\left(m^{3}\right)$} & & & & & & & & & & & & & & & & \\
\hline & \multicolumn{15}{|c|}{ dap (cm) } & \\
\hline $\mathrm{h}(\mathrm{m})$ & 10 & 11 & 12 & 13 & 14 & 15 & 16 & 17 & 18 & 19 & 20 & 21 & 22 & 23 & 24 & $h_{100}$ \\
\hline \multirow{2}{*}{10,9} & 0,0353 & 0,0424 & 0,0501 & 0,0585 & 0,0675 & 0,0771 & 0,0873 & 0,0981 & 0,1095 & 0,1216 & 0,1342 & 0,1474 & 0,1613 & 0,1757 & 0,1907 & \multirow{2}{*}{11,8} \\
\hline & 0,0286 & 0,0343 & 0,0406 & 0,0474 & 0,0547 & 0,0625 & 0,0708 & 0,0796 & 0,0889 & 0,0987 & 0,1090 & 0,1197 & 0,1310 & 0,1427 & 0,1549 & \\
\hline \multirow{2}{*}{13,3} & 0,0467 & 0,0562 & 0,0664 & 0,0775 & 0,0894 & 0,1021 & 0,1156 & 0,1300 & 0,1451 & 0,1610 & 0,1778 & 0,1953 & 0,2136 & 0,2327 & 0,2526 & \multirow{2}{*}{15,3} \\
\hline & 0,0382 & 0,0459 & 0,0543 & 0,0634 & 0,0731 & 0,0835 & 0,0946 & 0,1064 & 0,1188 & 0,1319 & 0,1456 & 0,1600 & 0,1750 & 0,1907 & 0,2071 & \\
\hline 15,5 & 0,0473 & 0,0568 & 0,0673 & 0,0785 & 0,0906 & 0,1035 & 0,1172 & 0,1318 & 0,1472 & 0,1634 & 0,1804 & 0,1982 & 0,2169 & 0,2363 & 0,2565 & 18,5 \\
\hline \multirow{2}{*}{17,5} & 0,0676 & 0,0813 & 0,0961 & 0,1122 & 0,1294 & 0,1478 & 0,1674 & 0,1881 & 0,2100 & 0,2331 & 0,2573 & 0,2827 & 0,3092 & 0,3369 & 0,3657 & \multirow{2}{*}{21,4} \\
\hline & 0,0559 & 0,0672 & 0,0795 & 0,0928 & 0,1071 & 0,1224 & 0,1387 & 0,1559 & 0,1741 & 0,1932 & 0,2134 & 0,2344 & 0,2565 & 0,2795 & 0,3034 & \\
\hline \multirow{2}{*}{19,4} & 0,0772 & 0,0928 & 0,1097 & 0,1280 & 0,1477 & 0,1687 & 0,1910 & 0,2147 & 0,2397 & 0,2660 & 0,2937 & 0,3226 & 0,3529 & 0,3845 & 0,4174 & \multirow{2}{*}{24,2} \\
\hline & 0,0641 & 0,0771 & 0,0912 & 0,1065 & 0,1228 & 0,1403 & 0,1590 & 0,1787 & 0,1996 & 0,2216 & 0,2446 & 0,2688 & 0,2941 & 0,3204 & 0,3479 & \\
\hline \multirow{2}{*}{21,1} & 0,0862 & 0,1036 & 0,1226 & 0,1430 & 0,1650 & 0,1884 & 0,2134 & 0,2398 & 0,2678 & 0,2972 & 0,3281 & 0,3604 & 0,3942 & 0,4295 & 0,4662 & \multirow{2}{*}{26,7} \\
\hline & 0,0719 & 0,0865 & 0,1023 & 0,1194 & 0,1378 & 0,1574 & 0,1783 & 0,2004 & 0,2238 & 0,2485 & 0,2743 & 0,3015 & 0,3298 & 0,3594 & 0,3901 & \\
\hline 22,7 & 0,0948 & 0,1139 & 0,1347 & 0,1572 & 0,1814 & 0,2072 & 0,2346 & 0,2637 & 0,2944 & 0,3267 & 0,3607 & 0,3962 & 0,4334 & 0,4722 & 0,5126 & 29,1 \\
\hline \multirow{2}{*}{24,3} & 0,1030 & 0,1237 & 0,1463 & 0,1707 & 0,1969 & 0,2250 & 0,2547 & 0,2863 & 0,3197 & 0,3548 & 0,3917 & 0,4303 & 0,4706 & 0,5127 & 0,5566 & \multirow{2}{*}{31,3} \\
\hline & 0,0864 & 0,1039 & 0,1229 & 0,1434 & 0,1655 & 0,1891 & 0,2142 & 0,2408 & 0,2689 & 0,2985 & 0,3296 & 0,3621 & 0,3962 & 0,4317 & 0,4687 & \\
\hline \multirow{2}{*}{25,7} & 0,1107 & 0,1330 & 0,1573 & 0,1836 & 0,2118 & 0,2419 & 0,2739 & 0,3079 & 0,3438 & 0,3815 & 0,4212 & 0,4627 & 0,5061 & 0,5514 & 0,5985 & \multirow{2}{*}{33,5} \\
\hline & 0,0931 & 0,1120 & 0,1325 & 0,1546 & 0,1784 & 0,2038 & 0,2309 & 0,2596 & 0,2899 & 0,3218 & 0,3553 & 0,3904 & 0,4271 & 0,4654 & 0,5053 & \\
\hline \multirow{2}{*}{27,1} & 0,1181 & 0,1419 & 0,1679 & 0,1959 & 0,2259 & 0,2581 & 0,2922 & 0,3285 & 0,3667 & 0,4070 & 0,4493 & 0,4936 & 0,5399 & 0,5882 & 0,6385 & \multirow{2}{*}{35,5} \\
\hline & 0,0996 & 0,1197 & 0,1416 & 0,1653 & 0,1908 & 0,2180 & 0,2469 & 0,2776 & 0,3100 & 0,3441 & 0,3799 & 0,4175 & 0,4567 & 0,4977 & 0,5403 & \\
\hline \multirow{2}{*}{28,4} & 0,1252 & 0,1504 & 0,1779 & 0,2076 & 0,2395 & 0,2735 & 0,3098 & 0,3482 & 0,3887 & 0,4314 & 0,4762 & 0,5232 & 0,5723 & 0,6235 & 0,6768 & \multirow{2}{*}{37,3} \\
\hline & 0,1058 & 0,1272 & 0,1505 & 0,1756 & 0,2026 & 0,2315 & 0,2622 & 0,2948 & 0,3292 & 0,3655 & 0,4035 & 0,4434 & 0,4851 & 0,5286 & 0,5739 & \\
\hline 296 & 0,1320 & 0,1586 & 0,1875 & 0,2188 & 0,2524 & 0,2883 & 0,3265 & 0,3670 & 0,4098 & 0,4548 & 0,5020 & 0,5515 & 0,6033 & 0,6572 & 0,7134 & 391 \\
\hline 23,0 & 0,1117 & 0,1343 & 0,1589 & 0,1855 & 0,2140 & 0,2445 & 0,2770 & 0,3114 & 0,3477 & 0,3860 & 0,4262 & 0,4683 & 0,5124 & 0,5583 & 0,6061 & (3), \\
\hline 308 & 0,1385 & 0,1664 & 0,1968 & 0,2296 & 0,2649 & 0,3026 & 0,3426 & 0,3851 & 0,4300 & 0,4772 & 0,5268 & 0,5787 & 0,6330 & 0,6896 & 0,7486 & 400 \\
\hline 30,8 & 0,1175 & 0,1412 & 0,1670 & 0,1950 & 0,2250 & 0,2570 & 0,2911 & 0,3273 & 0,3655 & 0,4058 & 0,4480 & 0,4923 & 0,5386 & 0,5869 & 0,6371 & 40,9 \\
\hline 20 & 0,1447 & 0,1739 & 0,2057 & 0,2400 & 0,2768 & 0,3162 & 0,3581 & 0,4025 & 0,4494 & 0,4987 & 0,5506 & 0,6049 & 0,6616 & 0,7208 & 0,7824 & 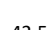 \\
\hline 31,9 & 0,1230 & 0,1478 & 0,1749 & 0,2041 & 0,2355 & 0,2691 & 0,3048 & 0,3427 & 0,3827 & 0,4248 & 0,4690 & 0,5154 & 0,5638 & 0,6144 & 0,6670 & 42,5 \\
\hline 330 & 0,1508 & 0,1812 & 0,2142 & 0,2500 & 0,2884 & 0,3294 & 0,3730 & 0,4193 & 0,4681 & 0,5195 & 0,5735 & 0,6300 & 0,6891 & 0,7508 & 0,8150 & 44 \\
\hline (3, & 0,1283 & 0,1542 & 0,1824 & 0,2129 & 0,2457 & 0,2807 & 0,3179 & 0,3574 & 0,3992 & 0,4431 & 0,4893 & 0,5376 & 0,5882 & 0,6409 & 0,6958 & 44,1 \\
\hline
\end{tabular}

\section{CONCLUSÕES}

Os três modelos ajustados e validados foram eficientes para estimar os volumes com e sem casca das árvores de $E$. grandis não havendo diferença estatística significativa entre suas estimativas e os volumes reais.

O modelo de Husch não leva em conta características de sítio, é bastante prático, mas em locais com condições diferentes da área de estudo pode não apresentar a mesma eficiência, sendo recomendado uma validação para averiguar a magnitude dos erros.

O modelo de Schumacher e Hall (modificado) apresentou os melhores resultados, sendo este composto por variáveis como diâmetro e altura total, corriqueiras nas estimativas de volume, além da altura dominante, variável que possibilita aplicação em outros locais que apresentem características de sítio semelhantes.

\section{REFERÊNCIAS}

ALVARES, C.A. et al. Köppen's climate classification map for Brazil. Meteorologische Zeitschrift, v.22, n.6, p.711-728, 2013.

AZEVEDO, T.L. et al. Equações hipsométricas e volumétricas para um povoamento de Eucalyptus sp. localizado na FLONA do IBURA, Sergipe. Revista Brasileira de Ciências Agrárias, v.6, n.1, p.105-
112,2011

AZEVEDO, G.B. et al. Estimativas volumétricas em povoamentos de eucalipto sob regime de alto fuste e talhadia no sudoeste da Bahia. Pesquisa florestal brasileira, v.31, n.68, p.309-31, 2011.

BINOTI, M.L. et al. Redes neurais artificiais para estimação do volume de árvores. Revista Árvore, v.38, n.2, p.283-288, 2014.

BUENO, G.F. et al. Modelagem linear generalizada para descrever a relação hipsométrica de Eucalytpus saligna Smith. Agrarian Academy, v.7, n.13, p.35-44, 2020.

CORDEIRO, M.A. et al. Estimativa do volume de Acacia mangium utilizando técnicas de redes neurais artificiais e máquinas vetor de suporte. Pesquisa Florestal Brasileira, v.35, n.83, p.255-261, 2015.

COUTO, H.T.Z.; BASTOS, N.L.M. Modelos de equações de volume e relações hipsométricas para plantações de Eucalyptus no estado de São Paulo. IPEF, n.37. p.33-44, 1987.

CUNHA, S.U. Dendrometria e inventário florestal. Manaus. Escola Agrotécnica Federal de Manaus, 2004.

EMBRAPA - Empresa Brasileira de Pesquisa Agropecuária. Sistema Brasileiro de Classificação de Solos. 5.ed. EMBRAPA, 2018.

FEPAM - Fundação Estadual de Proteção Ambiental Henrique Luis Roessler. Mapa de classificação dos solos do Estado do Rio 
Grande do Sul quanto à resistência a impactos ambientais. Porto Alegre: FEPAM, 2001.

FINGER, C.A.G. Fundamentos de biometria florestal. Santa Maria: UFSM/CEPEF/FATEC, 1992.

GAROSI, V.A. et al. Inventário florestal e recomendações de manejo para a floresta estadual de Assis - Instituto Florestal, SP. Revista científica eletrônica de engenharia florestal, v.7, n.12, p.1-27, 2008.

IBÁ - Indústria Brasileira de Árvores. Relatório 2019. IBÁ, 2019.

LANSSANOVA, L.R. et al. Comparação entre diferentes métodos para estimativa volumétrica de espécies comerciais da Amazônia. BIOFIX Scientific Journal, v.3, n.1, p.109-115, 2018.

LEAL, F.A. et al. Amostragem de árvores de Eucalyptus na cubagem rigorosa para estimativa de modelos volumétricos. Revista brasileira de biometria, v.33, n.1, p.91-103, 2015.

MARTINS, J.F.C. et al. Modelagem volumétrica de árvores de acácia-negra em povoamentos de regiões do Rio Grande do Sul. BIOFIX Scientific Journal, v.5, n.2, p.195-202, 2020.

MIGUEL, E.P. et al. Ajuste de modelo volumétrico e desenvolvimento de fator de forma para plantios de Eucalyptus grandis localizados no município de Rio Verde - GO. Enciclopédia Biosfera, v.6, n.11, p.1-13, 2010.

MIRANDA, D.L.C. et al. Fator de forma e equações de volume para estimativa volumétrica de árvores em plantio de Eucalyptus urograndis. Scientia Plena, v.11, n.3, p.1-8, 2015.

NETO, J.C.S. et al. Redes neurais artificiais na estimação de volume com casca de Mogno africano (Khaya ivorensis). Revista Brasileira de Biometria, v.37, n.2, p.191-204, 2019.

PEREIRA, A.R.S. et al. Modelagem volumétrica para Eucalyptus urograndis no Município de Porto Grande, Amapá, Brasil. Biota Amazônia, v.6, n.4, p.10-14, 2016.

OLIVEIRA, R.C.A. Equações volumétricas para Couratari stellata A. C. Smith (Tauari) na Floresta Nacional de Tapajós. Nativa, v.5, n.2, p.138-144, 2017.

R CORE TEAM. R: A language and environment for statistical computing. Vienna: R Foundation for Statistical Computing, 2016.

SALES, F.C.V. et al. Ajustes de modelos volumétricos para o clone de Eucalyptus grandis x Eucalyptus urophylla cultivados no agreste de Pernambuco. Floresta, v.45, n.4, p.663-670, 2015.

SANTOS, K.N.F. et al. Ajuste de equações volumétricas e redes neurais artificiais na estimativa do volume de Tauari na Floresta Nacional do Tapajós. Agroecossistemas, v.10, n.1, p.1-17, 2018.

SANQUETTA, C.R. et al. Estimativa da altura e do volume em povoamentos jovens de restauração florestal em Rondônia. BIOFIX Scientific Journal, v.2, n.2, p.23-31, 2017.

SILVA, R.C. et al. Ajuste de equações volumétricas para diferentes seccionamentos de tora em uma floresta sob manejo florestal comunitário. Acta Tecnológica, v.12, n.2, p.85-102, 2017.
SILVA, S. et al. Avaliação do uso de regressão e rede neural artificial para modelagem do afilamento do fuste de eucalipto em sistema silvipastoril. Enciclopédia Biosfera, v.13, n.23, p.189-199, 2016.

SOARES, C.P.B. et al. Dendrometria e inventário florestal. Viçosa: Ed. UFV, 2007.

STEPKA, T.F. et al. Modelos volumétricos e funções de afilamento para Pinus taeda L. na região Dos Campos Gerais, Paraná, Brasil. Revista Espacios, v.38, n.21, p.26, 2017.

TÉO, S.J. et al. Modelos de afilamento e forma dos fustes de eucalipto, em diferentes qualidades de sítio. Nativa, v.6, p.831841, 2018.

THOMAS, C. et al. Comparação de equações volumétricas ajustadas com dados de cubagem e análise de tronco. Ciência Florestal, v.16, n.3, p.319-327, 2006.

Recebido em 04-08-2020

Aceito em 05-10-2020 